\title{
The Medial Eminence Line for Predicting Tibial Fracture Risk after Unicompartmental Knee Arthroplasty
}

\author{
Ryo Yoshikawa, MD, Takafumi Hiranaka, MD, Koji Okamoto, MD, Takaaki Fujishiro, MD, \\ Yuichi Hida, MD, Tomoyuki Kamenaga, MD, Yoshitada Sakai, MD*
}

Department of Orthopedic Surgery and Joint Surgery Center, Takatsuki General Hospital, Osaka,

*Division of Rehabilitation Medicine, Kobe University Graduate School of Medicine, Kobe, Japan

Background: Tibial fracture after cementless Oxford unicompartmental knee arthroplasty (OUKA) is a rare but serious complication. It is reported more frequently in Asian countries. The aim of this retrospective study was to assess the morphological characteristics of the tibia by using a simple novel measurement method in patients with tibial fractures after OUKA.

Methods: Six knees (all women) with tibial fractures after cementless OUKA (fracture group) and 150 knees without an obvious complication after cementless OUKA (control group) were examined retrospectively at our institution between January 2016 and April 2017. We drew a medial eminence line (ME line) defined as a line extending from the tip of the medial intercondylar eminence parallel to the tibial axis. The ME line was classified into two types (intramedullary type [type I] and extramedullary type [type E]), and the proportion of each type was compared between fracture patients and controls.

Results: In the fracture group, there were four (66.7\%) type E cases and two (33.3\%) type I cases; in the control group, there were $18(12 \%)$ type $E$ cases and $132(88 \%)$ type I cases. Fisher exact tests showed that the proportion of type $E$ was higher in the fracture group than in the control group $(p<0.01)$.

Conclusions: Measurement of the ME line can be recommended as a useful method to assess the risk of postoperative fracture after cementless OUKA.

Keywords: Knee, Arthroplasty, Replacement, Unicompartmental knee arthroplasty, Tibial fracture

Unicompartmental knee arthroplasty (UKA) is a less invasive method for treating monocondylar arthritis of the knee than total knee arthroplasty (TKA). The use of UKA is gaining popularity and has shown excellent long-term results. ${ }^{1,2)}$ The survivorship of medial mobile bearing Oxford UKA (OUKA; Zimmer Biomet, Warsaw, IN, USA) is as high as $98 \%$ at 10 years ${ }^{3)}$ and $91 \%$ through the second

Received January 22, 2019; Accepted September 24, 2019

Correspondence to: Takafumi Hiranaka, MD

Department of Orthopedic Surgery and Joint Surgery Center, Takatsuki General Hospital, 1-3-13, Kosobe-Chou, Takatsuki-City, Osaka, 569-1192, Japan

Tel: +81-72-681-3801, Fax: +81-72-682-3834

E-mail: takafumi.hiranaka@gmail.com decade. ${ }^{4)}$

One of the most serious complications of UKA is tibial fracture. It has been described as a result of technical errors, such as the creation of an improper pin site for the fixation of the tibial cutting block, ${ }^{5)}$ vertical overcutting of the medial plateau, ${ }^{6}$ ) use of an excessive force with a heavy hammer, ${ }^{7)}$ and breach of the posterior tibial cortex during preparation of the tibial plateau for the implant. ${ }^{8)}$

Although rarely reported as a complication in Europe (less than $1 \%),{ }^{7,9,10)}$ tibial fracture is reported more frequently in Asian countries. ${ }^{11)}$ Yoshida et al. ${ }^{12)}$ described that the 10-year survival rate of $>1,000$ cases of OUKA in a Japanese population was $95.4 \%$, with good clinical results. However, fractures after UKA were reported to occur in $7.2 \%$ of cases in spite of good technical skill. ${ }^{13)}$ It may indi-

Copyright (C) 2020 by The Korean Orthopaedic Association 
Yoshikawa et al. Medial Eminence Line for Predicting Tibial Fracture after Unicompartmental Knee Arthroplasty

Clinics in Orthopedic Surgery • Vol. 12, No. 2, $2020 \bullet$ www.ecios.org

cate the influence of racial difference.

Studies have shown that Asian people have a varus knee alignment and Asian patients with varus knee osteoarthritis have a variable degree of tibia vara, a medial bow in their proximal tibia. ${ }^{14)}$ In proximal tibia vara, the tibial shaft offsets laterally from the center of the tibial plateau. ${ }^{15)}$ and the medial intercondylar eminence, which is the entry point of the vertical tibial cut during the OUKA procedure, is expected to be far from the tibial axis (TA). These conditions may be a possible risk factor for tibial fracture after UKA due to a lack of bone mass supporting tibial components where the total body weight load is concentrated.

Cementless fixation results in less frequent radiolucency than cemented fixation, as well as showing equivalent or superior clinical outcomes. ${ }^{10,16)}$ The removal of fragments of cement is particularly difficult with the minimally invasive surgery, leading to excessive wear and subsequent loosening of the implant. Although Liddle et al. ${ }^{17)}$ reported that tibial fracture was not frequent after 1,000 cementless UKAs, the occurrence of fracture itself is still a concern. It was reported that the cementless tibial component might increase the risk of perioperative tibial plateau fracture. ${ }^{18)}$

The purpose of this study was to confirm the relationship between the tibial fracture after cementless OUKA and the position of the medial intercondylar eminence in relation to the medial cortex of the tibial shaft. We hypothesized that the medial intercondylar eminence located medial to the cortex can be a risk factor for tibial fracture during UKA.
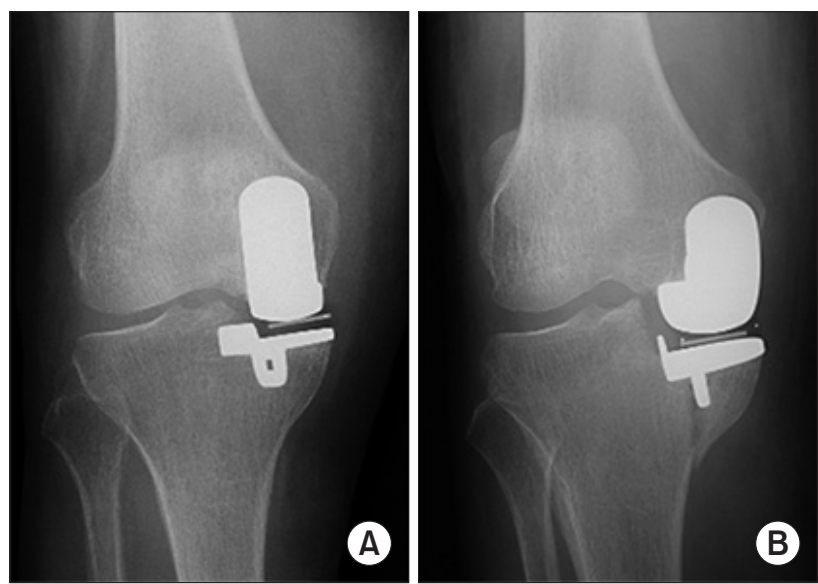

Fig. 1. (A) Immediate postoperative radiograph showing no fracture. (B) Two-week postoperative radiograph showing tibial plateau fracture.

\section{METHODS}

\section{Ethics Statement}

This study was approved by the Ethics Committee and Institutional Review Board of Takatsuki General Hospital (IRB No. 2018-79), and written informed consent was obtained from all patients.

\section{Patients}

Between January 2016 and April 2017, 156 cementless OUKAs were performed on 118 patients at Takatsuki General Hospital. There were six cases (fracture group) with a tibial fracture after operation (Fig. 1) and 150 cases (control group) without a fracture. In the fracture group, the six patients were all women, and the mean age at the time of operation was 76 years (range, 69-82 years). In the control group, there were 77 female and 35 male patients, and their mean age was 73 years (range, $47-90$ years) at the time of surgery.

\section{X-ray Assessment}

Anteroposterior knee X-ray images were evaluated retrospectively. Images with the patella not at the center of the femoral condyle were excluded. We defined the TA as a line passing through the center points at 6 and $12 \mathrm{~cm}$ below the joint line. The medial eminence line (ME line) was defined as a line running parallel to the TA and extending from the tip of the medial eminence (Fig. 2). We classified the knees into two categories based on the relationship between the ME line and the medial tibial cortex. The type E knee shows the ME line passing medial to the medial cortex (extramedullary), indicating a protrusion of the medial

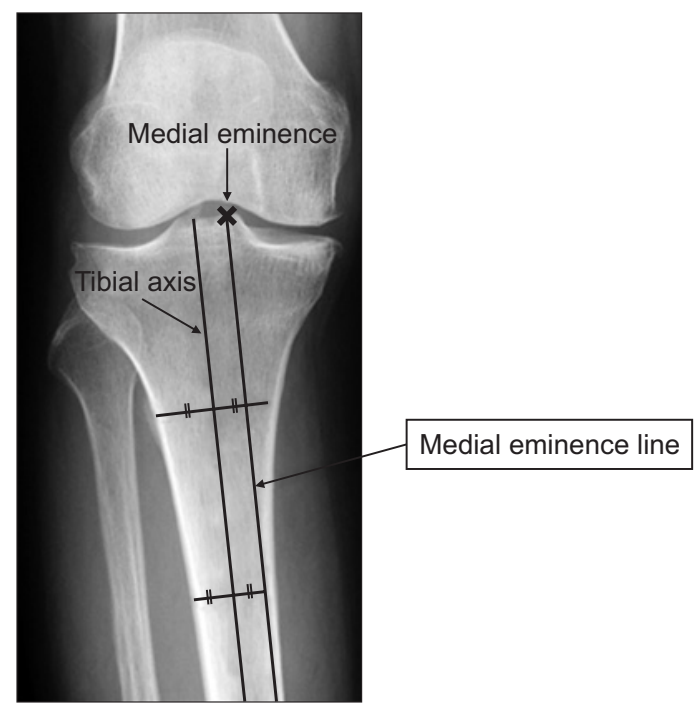

Fig. 2. Medial eminence line. 
Yoshikawa et al. Medial Eminence Line for Predicting Tibial Fracture after Unicompartmental Knee Arthroplasty

Clinics in Orthopedic Surgery • Vol. 12, No. 2, $2020 \bullet$ www.ecios.org

condyle (Fig. 3). The type I knee shows the ME line passing lateral to the medial cortex or on the cortex (intramedullary).

In addition, we measured the tibia vara angle (TVA) by using the anteroposterior radiographs of the whole lower extremity. The TVA was measured as the angle between the anatomic longitudinal axis of the tibia and the axis connecting the tibial plateau center and the ankle center as described by Mori et al. (Fig. 4). ${ }^{19)}$

\section{Statistics}

The measurement values were expressed as mean \pm standard deviation. Fisher exact tests were used to compare categorical variables between the groups. Mann-Whitney
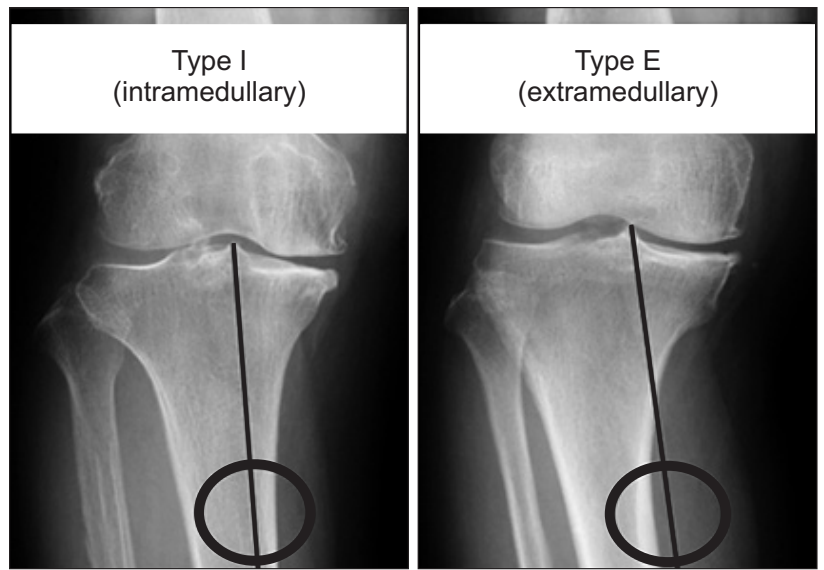

Fig. 3. Classification by medial eminence line.

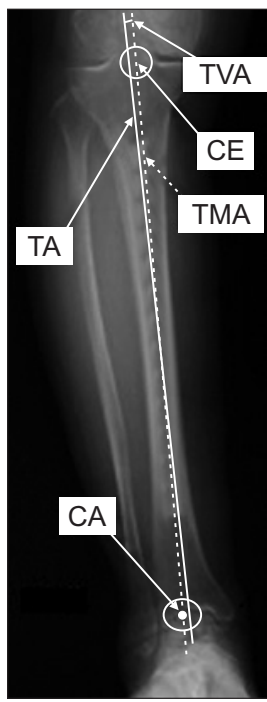

Fig. 4. Tibia vara angle (TVA). The tibial mechanical axis (TMA) is the axis connecting the center of the tibial eminence (CE) and the center of the ankle (CA). The TVA is the angle between the tibial axis (TA) and TMA.
$U$-tests were conducted to compare the fracture and ME line to TVA. Logistic regression was calculated to predict the fracture after OUKA based on sex, body mass index (BMI), age, TVA, and tibial morphology using the ME line. All data were analyzed by using IBM SPSS ver. 22.0 (IBM Japan, Tokyo, Japan). All analyses were performed by using $95 \%$ confidence intervals (CIs), and $p<0.05$ was considered statistically significant. Post-hoc power analysis was performed by using $G^{\star}$ Power $3 .{ }^{20)}$ For a sample size of 6 versus 150 elements in two groups and a type I error (a) of 0.05 (Fisher exact test), the study was expected to provide a power $(1-\beta)$ of 0.85 Hedges' $g$.

\section{RESULTS}

In the fracture group, there were four (66.7\%) type $\mathrm{E}$ cases and two (33.3\%) type I cases; in the control group, there were $18(12 \%)$ type E cases and $132(88 \%)$ type I cases (Table 1 ). The type E knee was significantly more common in the fracture group than in the control group (Fisher exact tests; odds ratio, 14.6; 95\% CI, 2.5 to 85.9 ; $p<0.05$; Hedges' $g=0.85$ ). Fisher exact tests showed no significant difference in the relationships between sex and ME line (Table 2). The average TVA of the control group and fracture group was $0.55^{\circ} \pm 0.49^{\circ}$ and $0.96^{\circ} \pm 0.25^{\circ}$, respectively (Mann-Whitney $U$-test, $p=0.018$; Hedges' g $=1.05$ ) (Fig. 5). The average TVA of type I and type $\mathrm{E}$ was $0.49^{\circ} \pm 0.45^{\circ}$ and $1.03^{\circ} \pm 0.43^{\circ}$, respectively (Mann-Whitney $U$-test, $p$ $<0.001$; Hedges' $g=1.25$ ) (Fig. 6). Although there were significant differences in both relations, the differences were less than $1.0^{\circ}$. Multiple logistic regression analysis for the occurrence of fracture was carried out with the following independent variables: sex, BMI, age, and ME line.

\begin{tabular}{l}
$\begin{array}{l}\text { Table 1. Relationship between Medial Eminence Line and the } \\
\text { Frequency of Fracture }\end{array}$ \\
\begin{tabular}{ccc} 
Variable & Type E & Type I \\
Fracture group & 4 & 2 \\
Control group & 18 & 132 \\
\hline$p<0.05$. &
\end{tabular} \\
\hline
\end{tabular}

Table 2. Relationship between Medial Eminence Line and Sex

\begin{tabular}{lcc} 
Variable & Type E & Type I \\
\hline Male & 3 & 42 \\
\hline Female & 19 & 92 \\
\hline
\end{tabular}

$p>0.05$. 
Yoshikawa et al. Medial Eminence Line for Predicting Tibial Fracture after Unicompartmental Knee Arthroplasty

Clinics in Orthopedic Surgery • Vol. 12, No. 2, $2020 \bullet$ www.ecios.org

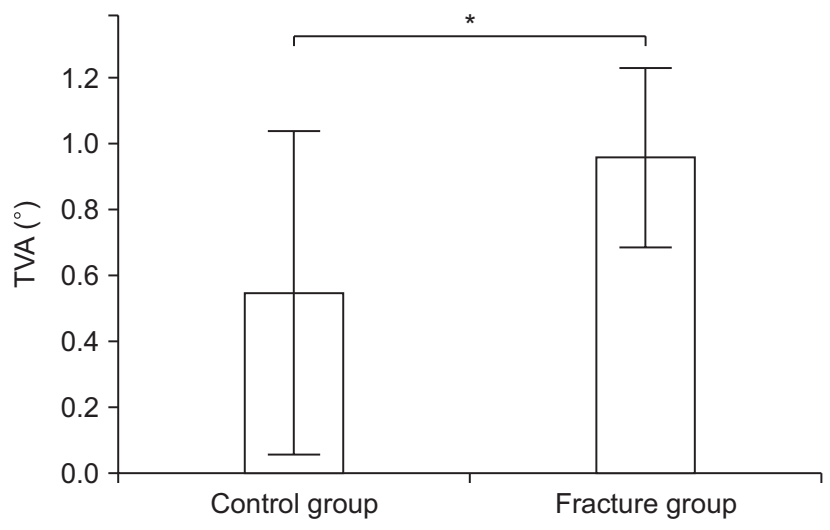

Fig. 5. Relationship between fracture and tibia vara angle (TVA). ${ }^{*} p<0.05$.

After eliminating interaction terms with a $p$-value greater than 0.05, BMI and ME line were retained in the model (Table 3). Patients with type E knees had a significantly higher risk of fracture than patients with type I knees (odds ratio, 14.0; 95\% CI, 2.33 to $83.74 ; p<0.01$ ). Patients with a low BMI had a significantly higher risk of fracture than patients with a high BMI (odds ratio, 0.84; $95 \% \mathrm{CI}, 0.79$ to $0.89 ; p<0.001)$.

\section{DISCUSSION}

Our results suggest that a patient with the type E knee has an increased risk of tibial fracture after cementless OUKA. The type E tibia is supposed to have a tibia vara where less bone volume supports the tibial component than in the type I knee. In addition, the distance from the keel to the medial cortex might be shorter in such patients. Because the keel of cementless OUKA is wider than the size of bone cavity, the force required to seat the component spreads on the tibial bone and can cause a fractures if the distance from the tip of keel to the tibial cortex is short. ${ }^{18)}$

Measuring TVA may predict the risk of fracture, but it is difficult to determine the cutoff value because it is a quantitative variable. The ME line is a qualitative variable and can be divided into two categories. It is a simple method to predict the risk of fracture after UKA. Because patients with an extramedullary ME line have a high risk of fracture, fractures can be reduced by the appropriate selection of cemented UKA or TKA for their treatment.

In the present study, although sex was eliminated by multiple logistic regression analysis, we had no male patients with tibial fractures. From this observation, we suggest that bone density and tibia size may impact the frequency of fracture. Female tibial fractures after UKA have been reported to occur more frequently because the bone density of the medial tibial plateau, which supports tibial

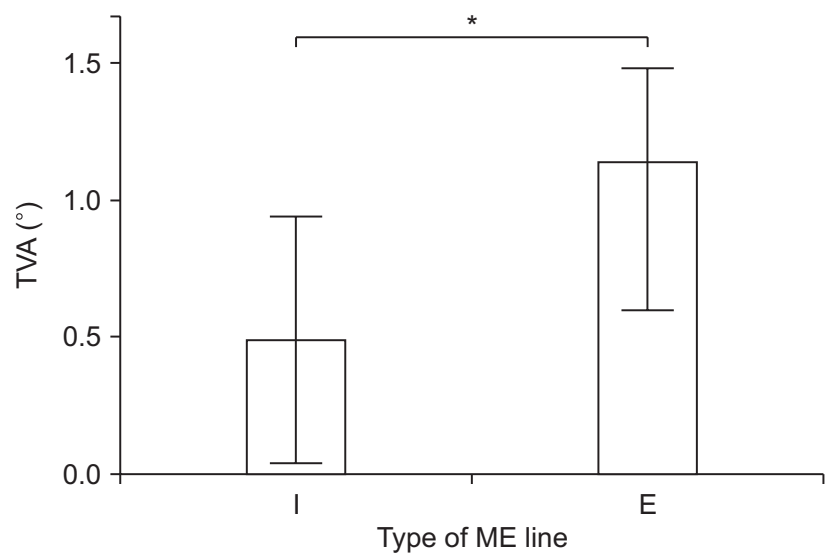

Fig. 6. Relationship between medial eminence line (ME line) and tibia vara angle (TVA). ${ }^{*} p<0.001$.

$\begin{aligned} & \text { Table 3. Multiple Logistic } \\
& \text { after OUKA }\end{aligned}$
\begin{tabular}{ccc}
\hline \multicolumn{4}{c}{ Variable } & Odds ratio $(95 \% \mathrm{CI})$ & $p$-value \\
\hline Medial eminence line & $13.964(2.329-83.739)$ & 0.004 \\
\hline Body mass index & $0.842(0.793-0.893)$ & $<0.001$ \\
\hline OUKA: Oxford unicompartmental knee arthroplasty, Cl: confidence Interval.
\end{tabular}

components, is less than that of male bone density and because the medial and lateral widths are smaller in women than in men. ${ }^{15)}$ Logistic regression analysis indicated that patients with a low BMI showed a high risk of fracture. Glogowska-Szelag ${ }^{21)}$ demonstrated that an increase in BMI was accompanied by an increase in bone mineral density (BMD). In this study, we did not examine BMD, but this correlation between BMI and BMD may explain our results.

The present study has several limitations. First, the number of patients with tibial fracture included in the study was small. Tibial fracture is a rare complication, and we should increase the number of cases examined by conducting a multicenter study. Second, we examined only Japanese patients. Evaluation of the ME line in Europe and other regions where tibial fracture occurs less frequently is important for comparison with our results in the Japanese population. Finally, the effect of the rotation of X-ray was not taken into account in this study. To adjust for this, three-dimensional computed tomographic analysis may be required.

Despite these limitations, ME line measurement can be valuable clinically because it is a simple method that can be applied universally. Using this method, surgeons may estimate the risk of postoperative fracture after UKA 
Yoshikawa et al. Medial Eminence Line for Predicting Tibial Fracture after Unicompartmental Knee Arthroplasty

Clinics in Orthopedic Surgery • Vol. 12, No. 2, $2020 \bullet$ www.ecios.org

and make informed decisions on the type of component to be used. Measurement of the ME line can be recommended as a considerable method to assess the risk of postoperative fracture after cementless OUKA.

\section{CONFLICT OF INTEREST}

No potential conflict of interest relevant to this article was reported.

\section{REFERENCES}

1. Berger RA, Nedeff DD, Barden RM, et al. Unicompartmental knee arthroplasty: clinical experience at 6- to 10-year followup. Clin Orthop Relat Res. 1999;(367):50-60.

2. Bergeson AG, Berend KR, Lombardi AV Jr, Hurst JM, Morris MJ, Sneller MA. Medial mobile bearing unicompartmental knee arthroplasty: early survivorship and analysis of failures in 1000 consecutive cases. J Arthroplasty. 2013;28(9 Suppl):172-5.

3. Murray DW, Goodfellow JW, O'Connor JJ. The Oxford medial unicompartmental arthroplasty: a ten-year survival study. J Bone Joint Surg Br. 1998;80(6):983-9.

4. Price AJ, Svard U. A second decade lifetable survival analysis of the Oxford unicompartmental knee arthroplasty. Clin Orthop Relat Res. 2011;469(1):174-9.

5. Brumby SA, Carrington R, Zayontz S, Reish T, Scott RD. Tibial plateau stress fracture: a complication of unicompartmental knee arthroplasty using 4 guide pinholes. J Arthroplasty. 2003;18(6):809-12.

6. Clarius M, Haas D, Aldinger PR, Jaeger S, Jakubowitz E, Seeger JB. Periprosthetic tibial fractures in unicompartmental knee arthroplasty as a function of extended sagittal saw cuts: an experimental study. Knee. 2010;17(1):57-60.

7. Pandit H, Murray DW, Dodd CA, et al. Medial tibial plateau fracture and the Oxford unicompartmental knee. Orthopedics. 2007;30(5 Suppl):28-31.

8. Sloper PJ, Hing CB, Donell ST, Glasgow MM. Intra-operative tibial plateau fracture during unicompartmental knee replacement: a case report. Knee. 2003;10(4):367-9.

9. Berger RA, Meneghini RM, Jacobs JJ, et al. Results of unicompartmental knee arthroplasty at a minimum of ten years of follow-up. J Bone Joint Surg Am. 2005;87(5):999-1006.

10. Akan B, Karaguven D, Guclu B, et al. Cemented versus uncemented Oxford unicompartmental knee arthroplasty: is there a difference? Adv Orthop. 2013;2013:245915.

11. Song MH, Kim BH, Ahn SJ, Yoo SH, Lee MS. Early complications after minimally invasive mobile-bearing medial unicompartmental knee arthroplasty. J Arthroplasty.
2009;24(8):1281-4.

12. Yoshida K, Tada M, Yoshida H, Takei S, Fukuoka S, Nakamura H. Oxford phase 3 unicompartmental knee arthroplasty in Japan: clinical results in greater than one thousand cases over ten years. J Arthroplasty. 2013;28(9 Suppl):168-71.

13. Yokoyama M, Nakamura Y, Egusa M, et al. Factors related to stress fracture after unicompartmental knee arthroplasty. Asia Pac J Sports Med Arthrosc Rehabil Technol. 2019;15:15.

14. Hovinga KR, Lerner AL. Anatomic variations between Japanese and Caucasian populations in the healthy young adult knee joint. J Orthop Res. 2009;27(9):1191-6.

15. Nagamine $\mathrm{R}$, Miura $\mathrm{H}$, Bravo CV, et al. Anatomic variations should be considered in total knee arthroplasty. J Orthop Sci. 2000;5(3):232-7.

16. Pandit $\mathrm{H}$, Liddle $\mathrm{AD}$, Kendrick BJ, et al. Improved fixation in cementless unicompartmental knee replacement: fiveyear results of a randomized controlled trial. J Bone Joint Surg Am. 2013;95(15):1365-72.

17. Liddle AD, Pandit H, O’Brien S, et al. Cementless fixation in Oxford unicompartmental knee replacement: a multicentre study of 1000 knees. Bone Joint J. 2013;95(2):181-7.

18. Campi S, Mellon SJ, Ridley D, et al. Optimal interference of the tibial component of the cementless Oxford unicompartmental knee replacement. Bone Joint Res. 2018;7(3):226-31.

19. Mori S, Akagi M, Asada S, Matsushita T, Hashimoto K. Tibia vara affects the aspect ratio of tibial resected surface in female Japanese patients undergoing TKA. Clin Orthop Relat Res. 2013;471(5):1465-71.

20. Faul F, Erdfelder E, Buchner A, Lang AG. Statistical power analyses using $G^{\star}$ Power 3.1: tests for correlation and regression analyses. Behav Res Methods. 2009;41(4):1149-60.

21. Glogowska-Szelag J. Assessment of the relationship between bmd and body mass index BMI in women with postmenopausal osteoporosis. Wiad Lek. 2018;71(9):1714-8. 